International Journal of Pure and Applied Mathematics

Volume 109 No. 3 2016, 493-508

ISSN: 1311-8080 (printed version); ISSN: 1314-3395 (on-line version)

url: http://www.ijpam.eu

doi: 10.12732/ijpam.v109i3.2

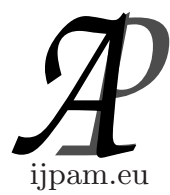

\title{
SOME DEGREE BASED TOPOLOGICAL INDICES \\ OF GENERALIZED TRANSFORMATION GRAPHS AND OF THEIR COMPLEMENTS
}

\author{
Harishchandra S. Ramane ${ }^{1}$, Raju B. Jummannaver ${ }^{2}$, Shaban Sedghi ${ }^{3}$ \\ ${ }^{1,2}$ Department of Mathematics \\ Karnatak University \\ Dharwad, 580003, Karnataka, INDIA \\ ${ }^{3}$ Department of Mathematics \\ Qaemshahr Branch \\ Islamic Azad University \\ Qaemshahr, IRAN
}

\begin{abstract}
In this paper, the expressions for the Geometric-arithmetic index, Sum-connectivity index, Atom-bond connectivity index and Augmented Zagreb index of the generalized transformation graphs and of their complement graphs are obtained.
\end{abstract}

AMS Subject Classification: 05C76, 05C07, 92E10

Key Words: geometric-arithmetic index, sum-connectivity index, atom-bond connectivity index, augmented Zagreb index, generalized transformation graphs

\section{Introduction}

The topological indices play an importatnt role in the studies of quantitative structure property relationship (QSPR) and quantitative structre activity relationship (QSAR) in mathematical chemistry. More studied topological indices based on the degree of a vertices are Randic index $[9,14]$, harmonic index $[5,10]$ and Zagreb index $[2,7,8]$.

Received: $\quad$ March 13, 2016

Revised: $\quad$ May 27, 2016

Published: September 27, 2016

$\S_{\text {Correspondence author }}$ (c) 2016 Academic Publications, Ltd. url: www.acadpubl.eu 
In [13] the harmonic index and Randic index of generalized transformation graphs were obtained. In this paper we obtain the other topological indices based on the degrees of the vertices such as geometric-arithmetic (GA) index, sum connectivity (SC) index, atom bond connectivity (ABC) index and augmented Zagreb (AZ) index of generalized transformation graphs.

Let $G$ be a simple, undirected graph with $n$ verticses and $m$ edges. Let $V(G)$ and $E(G)$ be the vertex set and edge set of $G$ respectively. If $u$ and $v$ are adjacent vertices of $G$, then the edge connecting them will be denoted by $u v$. The degree of a vertex $v$ in $G$ is the number of edges incident to it and is denoted by $d_{G}(v)$.

The geometric-arithmetic index is defined as

$$
G A(G)=\sum_{u v \in E(G)} \frac{\sqrt{\left(d_{G}(u)\right)\left(d_{G}(v)\right)}}{\frac{1}{2}\left[d_{G}(u)+d_{G}(v)\right]} .
$$

This index was invented by Vukičević and Furtula [16].

The sum-connectivity index of a graph $G$ is defined as

$$
S C(G)=\sum_{u v \in E(G)} \frac{1}{\sqrt{d_{G}(u)+d_{G}(v)}} .
$$

It was proposed in [17] and it is found that the sum-connectivity index and the product connectivity index (that is, Randic index) correlate well among themselves and with the $\pi$-electronic energy of benzenoid hydrocarbons [12]. More applications of the sum-connectivity index may be found in [11].

Estrada et al. [4] proposed a topological index called atom-bond connectivity index. It is defined as

$$
A B C(G)=\sum_{u v \in E(G)} \sqrt{\frac{d_{G}(u)+d_{G}(v)-2}{d_{G}(u) d_{G}(v)}} .
$$

This index has proven to be a valuable predictive index in the study of the formation of heat in alkanes [4]. Estrada [3] developed a basically topological approach on the basis of the ABC index which explains the differences in the energy of linear and branched alkanes both qualitatively and quantitatively.

The augmented Zagreb index of a graph $G$, proposed by Furtula et al. [6], is defined as

$$
A Z(G)=\sum_{u v \in E(G)}\left[\frac{d_{G}(u) d_{G}(v)}{d_{G}(u)+d_{G}(v)-2}\right]^{3} .
$$


It has been shown that $\mathrm{AZ}$ index is a valuable predictive index in the study of the heat of formation in octanes and heptanes [6], whose prediction power is better than $\mathrm{ABC}$ index.

In [13], the harmonic index and Randić index of generalized transformation graphs were obtained. Knowing the degrees of the vertices of $G$, one can get the degree based topological indices, but for a large non-regular structure it is less trivial to obtain such topological indices. Using the technique of [13], in this paper we obtain the expressions for the Geometric-Arithmetic Index, SumConnectivity Index, Atom-Bond Connectivity Index and Augmented Zagreb Index of generalized transformation graphs $G^{x y}$ and of their complements $\overline{G^{x y}}$ in terms of the parameters of underlying graph $G$.

\section{Generalized Transformation Graphs $G^{x y}$}

Let $G$ be a graph with vertex set $V(G)$ and edge set $E(G)$. The generalized transformation graph [1] of a graph $G$, denoted by $G^{x y}$, is a graph whose vertex set is $V(G) \cup E(G)$, and $\alpha, \beta \in V\left(G^{x y}\right)$. The vertices $\alpha$ and $\beta$ are adjacent in $G^{x y}$ if and only if (a) and (b) holds:

(a) $\alpha, \beta \in V(G), \alpha, \beta$ are adjacent in $G$ if $x=+$ and $\alpha, \beta$ are not adjacent in $G$ if $x=-$.

(b) $\alpha \in V(G)$ and $\beta \in E(G), \alpha, \beta$ are incident in $G$ if $y=+$ and $\alpha, \beta$ are not incident in $G$ if $y=-$.

There are four graphical transformations of a graph, such as $G^{++}, G^{+-}$, $G^{-+}$and $G^{--}$. The transformation $G^{++}$of $G$ is a semitotal-point graph defined in [15]. An example of generalized graph transformations and their complements are depicted in the Figure 1.

The vertex $v$ of $G^{x y}$ corresponding to a vertex $v$ of $G$ is referred to as a point vertex. The vertex $e$ of $G^{x y}$ corresponding to an edge $e$ of $G$ is referred to as a line vertex.

Proposition 2.1. [1] Let $G$ be a graph with $n$ vertices and $m$ edges. Let $u \in V(G)$ and $e \in E(G)$. Then the degrees of point and line vertices in $G^{x y}$ are (i) $d_{G^{++}}(u)=2 d_{G}(u)$ and $d_{G^{++}}(e)=2$.

(ii) $d_{G^{+-}}(u)=m$ and $d_{G^{+-}}(e)=n-2$.

(iii) $d_{G^{-+}}(u)=n-1$ and $d_{G^{-+}}(e)=2$.

(iv) $d_{G^{--}}(u)=n+m-1-2 d_{G}(u)$ and $d_{G^{--}}(e)=n-2$.

The complement of $G$ will be denoted by $\bar{G}$.

Proposition 2.2. [13] Let $G$ be a graph with $n$ vertices and $m$ edges. Let 

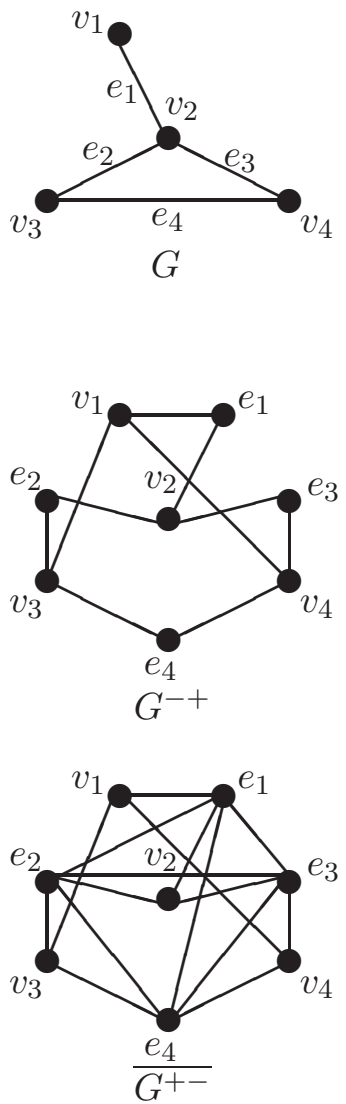
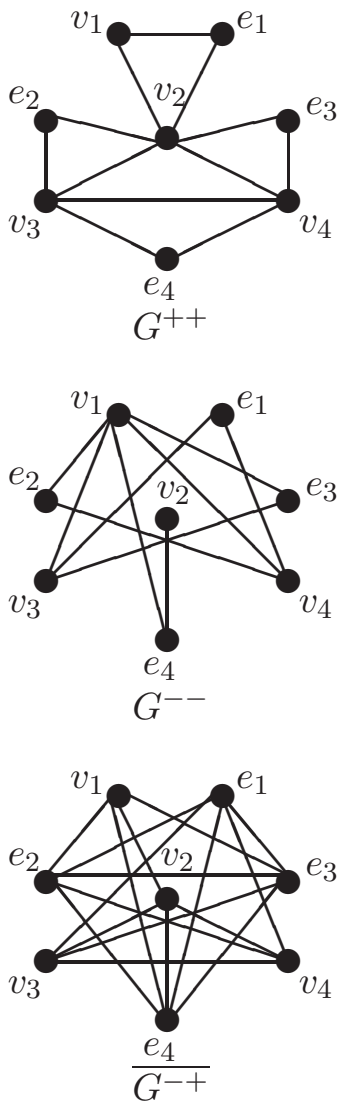
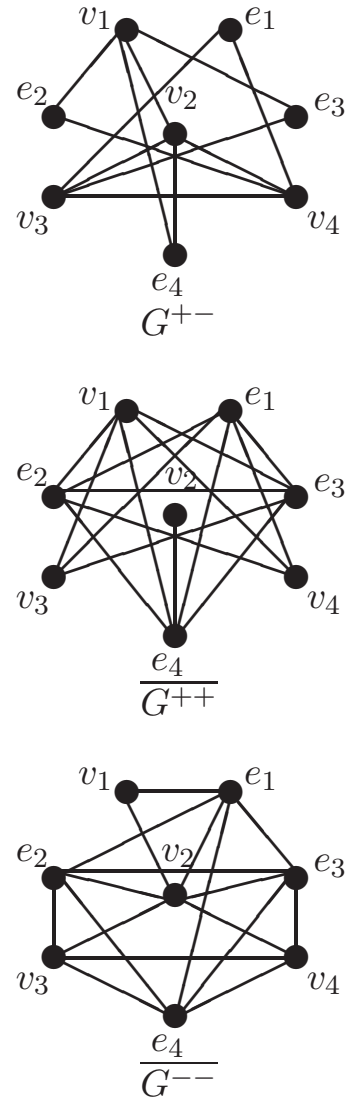

Figure 1: Graph, its generalized transformations and their complements

$u \in V(G)$ and $e \in E(G)$. Then the degrees of point and line vertices in $\overline{G^{x y}}$ are

(i) $d \overline{G^{++}}(u)=n+m-1-2 d_{G}(u)$ and $d_{\overline{G^{++}}}(e)=n+m-3$.

(ii) $d \overline{G^{+-}}(u)=n-1$ and $d_{\overline{G^{+-}}}(e)=m+1$.

(iii) $d_{\overline{G^{-+}}}(u)=m$ and $d_{\overline{G^{-+}}}(e)=n+m-3$.

(iv) $d \overline{G^{--}}(u)=2 d_{G}(u)$ and $d \overline{G^{--}}(e)=m+1$. 


\section{Geometric-Arithmetic Index of $G^{x y}$}

Theorem 3.1. Let $G$ be a graph with $n$ vertices and $m$ edges. Then

$$
G A\left(G^{++}\right)=G A(G)+\sum_{u \in V(G)} \frac{2\left(d_{G}(u)\right)^{3 / 2}}{1+d_{G}(u)} .
$$

Proof. Partition the edge set $E\left(G^{++}\right)$into two sets $E_{1}$ and $E_{2}$, where $E_{1}=$ $\{u v \mid u v \in E(G)\}$ and $E_{2}=\{u e \mid$ the vertex $u$ is incident to the edge $e$ in $G\}$. It is easy to check that $\left|E_{1}\right|=m$ and $\left|E_{2}\right|=2 m$. By Proposition 2.1, if $u \in V(G)$ then $d_{G^{++}}(u)=2 d_{G}(u)$ and if $e \in E(G)$ then $d_{G^{++}}(e)=2$.

Therefore

$$
\begin{aligned}
G A\left(G^{++}\right) & =\sum_{u v \in E\left(G^{++}\right)} \frac{\sqrt{d_{G^{++}}(u) d_{G^{++}}(v)}}{\frac{1}{2}\left[d_{G^{++}}(u)+d_{G^{++}}(v)\right]} \\
& =\sum_{u v \in E_{1}} \frac{\sqrt{d_{G^{+}}(u) d_{G^{++}}(v)}}{\frac{1}{2}\left[d_{G^{++}}(u)+d_{G^{++}}(v)\right]}+\sum_{u e \in E_{2}} \frac{\sqrt{d_{G^{++}}(u) d_{G^{++}}(e)}}{\frac{1}{2}\left[d_{G^{++}}(u)+d_{G^{++}}(e)\right]} \\
& =\sum_{u v \in E(G)} \frac{\sqrt{2 d_{G}(u) 2 d_{G}(v)}}{\frac{1}{2}\left[2 d_{G}(u)+2 d_{G}(v)\right]}+\sum_{u e \in E_{2}} \frac{\sqrt{2 d_{G}(u) 2}}{\frac{1}{2}\left[2 d_{G}(u)+2\right]} \\
& =\sum_{u v \in E(G)} \frac{\sqrt{d_{G}(u) d_{G}(v)}}{\frac{1}{2}\left[d_{G}(u)+d_{G}(v)\right]}+\sum_{u e \in E_{2}} \frac{2 \sqrt{d_{G}(u)}}{d_{G}(u)+1} \\
& =G A(G)+\sum_{u e \in E_{2}} \frac{2 \sqrt{d_{G}(u)}}{1+d_{G}(u)}
\end{aligned}
$$

In the second part of above equation, the quantity $\frac{\sqrt{d_{G}(u)}}{1+d_{G}(u)}$ appears $d_{G}(u)$ times. Hence above expression can be written as

$$
G A\left(G^{++}\right)=G A(G)+\sum_{u \in V(G)} \frac{2\left(d_{G}(u)\right)^{3 / 2}}{1+d_{G}(u)} .
$$

Theorem 3.2. Let $G$ be a graph with $n$ vertices and $m$ edges. Then

$$
G A\left(G^{+-}\right)=m+\frac{2 \sqrt{[m(n-2)]^{3}}}{(n-2+m)} .
$$


Proof. Partition the edge set $E\left(G^{+-}\right)$into two sets $E_{1}$ and $E_{2}$, where $E_{1}=$ $\{u v \mid u v \in E(G)\}$ and

$$
E_{2}=\{u e \mid \text { the vertex } u \text { is not incident to the edge } e \text { in } G\} \text {. }
$$

It is easy to check that $\left|E_{1}\right|=m$ and $\left|E_{2}\right|=m(n-2)$. By Proposition 2.1, if $u \in V(G)$ then $d_{G^{+-}}(u)=m$ and if $e \in E(G)$ then $d_{G^{+-}}(e)=n-2$.

Therefore

$$
\begin{aligned}
G A\left(G^{+-}\right) & =\sum_{u v \in E\left(G^{+-}\right)} \frac{\sqrt{d_{G^{+-}}(u) d_{G^{+-}}(v)}}{\frac{1}{2}\left[d_{G^{+-}}(u)+d_{G^{+-}}(v)\right]} \\
& =\sum_{u v \in E_{1}} \frac{\sqrt{d_{G^{+-}}(u) d_{G^{+-}}(v)}}{\frac{1}{2}\left[d_{G^{+-}}(u)+d_{G^{+-}}(v)\right]}+\sum_{u e \in E_{2}} \frac{\sqrt{d_{G^{+-}}(u) d_{G^{+-}}(e)}}{\frac{1}{2}\left[d_{G^{+-}}(u)+d_{G^{+-}}(e)\right]} \\
& =\sum_{u v \in E(G)} \frac{\sqrt{(m)(m)}}{\frac{1}{2}[m+m]}+\sum_{u e \in E_{2}} \frac{\sqrt{m(n-2)}}{\frac{1}{2}[m+n-2]} \\
& =\frac{m \sqrt{m^{2}}}{\frac{1}{2}(2 m)}+\frac{m(n-2)}{\frac{1}{2}(n-2+2)} \\
& =m+\frac{2 \sqrt{[m(n-2)]^{3}}}{(n-2+m)}
\end{aligned}
$$

Theorem 3.3. Let $G$ be a graph with $n$ vertices and $m$ edges. Then

$$
G A\left(G^{-+}\right)=\frac{n(n-1)-2 m}{2}+\frac{4 m \sqrt{2(n-1)}}{n+1} .
$$

Proof. Partition the edge set $E\left(G^{-+}\right)$into two sets $E_{1}$ and $E_{2}$, where $E_{1}=$ $\{u v \mid u v \notin E(G)\}$ and $E_{2}=\{u e \mid$ the vertex $u$ is incident to the edge $e$ in $G\}$. It is easy to check that $\left|E_{1}\right|=\left(\begin{array}{c}n \\ 2\end{array}\right)-m$ and $\left|E_{2}\right|=2 m$. By Proposition 2.1, if $u \in V(G)$ then $d_{G^{-+}}(u)=n-1$ and if $e \in E(G)$ then $d_{G^{-+}}(e)=2$.

Therefore

$$
\begin{aligned}
G A\left(G^{-+}\right) & =\sum_{u v \in E\left(G^{-+}\right)} \frac{\sqrt{d_{G^{-+}}(u) d_{G^{-+}}(v)}}{\frac{1}{2}\left[d_{G^{-+}}(u)+d_{G^{-+}}(v)\right]} \\
& =\sum_{u v \in E_{1}} \frac{\sqrt{d_{G^{-+}}(u) d_{G^{-+}}(v)}}{\frac{1}{2}\left[d_{G^{-+}}(u)+d_{G^{-+}}(v)\right]}+\sum_{u e \in E_{2}} \frac{\sqrt{d_{G^{-+}}(u) d_{G^{-+}}(e)}}{\frac{1}{2}\left[d_{G^{-+}}(u)+d_{G^{-+}}(e)\right]} \\
& =\sum_{u v \notin E(G)} \frac{\sqrt{(n-1)(n-1)}}{\frac{1}{2}(n-1+n-1)}+\sum_{u e \in E_{2}} \frac{\sqrt{2(n-1)}}{\frac{1}{2}(n-1+2)}
\end{aligned}
$$




$$
\begin{aligned}
& =\left[\left(\begin{array}{l}
n \\
2
\end{array}\right)-m\right] \frac{\sqrt{(n-1)^{2}}}{\frac{1}{2}[2(n-1)]}+\frac{2 m \sqrt{2(n-1)}}{\frac{1}{2}(n-1+2)} \\
& =\frac{n(n-1)-2 m}{2}+\frac{4 m \sqrt{2(n-1)}}{n+1}
\end{aligned}
$$

Theorem 3.4. Let $G$ be a graph with $n$ vertices and $m$ edges. Then

$$
\begin{aligned}
G A\left(G^{--}\right)= & \sum_{u v \notin E(G)} \frac{\sqrt{\left(n+m-1-2 d_{G}(u)\right)\left(n+m-1-2 d_{G}(v)\right)}}{n+m-1-\left(d_{G}(u)+d_{G}(v)\right)} \\
& +\sum_{u \in V(G)} \frac{\left(m-d_{G}(u)\right) \sqrt{\left(n+m-1-2 d_{G}(u)\right)(n-2)}}{\frac{1}{2}\left[2 n+m-3-2 d_{G}(u)\right]}
\end{aligned}
$$

Proof. Partition the edge set $E\left(G^{--}\right)$into two sets $E_{1}$ and $E_{2}$, where $E_{1}=$ $\{u v \mid u v \notin E(G)\}$ and

$$
E_{2}=\{u e \mid \text { the vertex } u \text { is not incident to the edge } e \text { in } G\} .
$$

It is easy to check that $\left|E_{1}\right|=\left(\begin{array}{l}n \\ 2\end{array}\right)-m$ and $\left|E_{2}\right|=m(n-2)$. By Proposition 2.1, if $u \in V(G)$ then $d_{G^{--}}(u)=n+m-1-2 d_{G}(u)$ and if $e \in E(G)$ then $d_{G^{--}}(e)=n-2$.

Therefore

$$
\begin{aligned}
G A\left(G^{--}\right) & =\sum_{u v \in E\left(G^{--}\right)} \frac{\sqrt{d_{G^{--}}(u) d_{G^{--}}(v)}}{\frac{1}{2}\left[d_{G^{--}}(u)+d_{G^{--}}(v)\right]} \\
& =\sum_{u v \in E_{1}} \frac{\sqrt{d_{G^{--}}(u) d_{G^{--}}(v)}}{\frac{1}{2}\left[d_{G^{--}}(u)+d_{G^{--}}(v)\right]}+\sum_{u e \in E_{2}} \frac{\sqrt{d_{G^{--}}(u) d_{G^{--}}(e)}}{\frac{1}{2}\left[d_{G^{--}}(u)+d_{G^{--}}(e)\right]} \\
& =\sum_{u v \notin E(G)} \frac{\sqrt{\left(n+m-1-2 d_{G}(u)\right)\left(n+m-1-2 d_{G}(v)\right)}}{\frac{1}{2}\left[n+m-1-2 d_{G}(u)+n+m-1-2 d_{G}(v)\right]} \\
& +\sum_{u e \in E_{2}} \frac{\sqrt{\left(n+m-1-2 d_{G}(u)\right)(n-2)}}{\frac{1}{2}\left[n+m-1-2 d_{G}(u)+n-2\right]} \\
& =\sum_{u v \notin E(G)} \frac{\sqrt{\left(n+m-1-2 d_{G}(u)\right)\left(n+m-1-2 d_{G}(v)\right)}}{n+m-1-\left(d_{G}(u)+d_{G}(v)\right)} \\
& +\sum_{u e \in E_{2}} \frac{\sqrt{\left(n+m-1-2 d_{G}(u)\right)(n-2)}}{\frac{1}{2}\left[2 n+m-3-2 d_{G}(u)\right]}
\end{aligned}
$$




$$
\begin{aligned}
& =\sum_{u v \notin E(G)} \frac{\sqrt{\left(n+m-1-2 d_{G}(u)\right)\left(n+m-1-2 d_{G}(v)\right)}}{n+m-1-\left(d_{G}(u)+d_{G}(v)\right)} \\
& +\sum_{u \in V(G)} \frac{\left(m-d_{G}(u)\right) \sqrt{\left(n+m-1-2 d_{G}(u)\right)(n-2)}}{\frac{1}{2}\left[2 n+m-3-2 d_{G}(u)\right]} .
\end{aligned}
$$

By Theorems 3.2 and 3.3 we have following corollary.

Corollary 3.5. Let $G_{1}$ and $G_{2}$ be two different graphs having same number of vertices and same number of edges. Then

(i) $G A\left(G_{1}^{+-}\right)=G A\left(G_{2}^{+-}\right)$

(ii) $G A\left(G_{1}^{-+}\right)=G A\left(G_{2}^{-+}\right)$.

\section{Geometric-Arithmetic Index of $\overline{G^{x y}}$}

Theorem 4.1. Let $G$ be a graph with $n$ vertices and $m$ edges. Then

$$
\begin{aligned}
G A\left(\overline{G^{++}}\right)= & \sum_{u v \notin E(G)} \frac{\sqrt{\left(n+m-1-2 d_{G}(u)\right)\left(n+m-1-2 d_{G}(v)\right)}}{n+m-1-\left(d_{G}(u)+d_{G}(v)\right)} \\
& +\sum_{u \in V(G)} \frac{\left(m-d_{G}(u)\right) \sqrt{\left(n+m-1-2 d_{G}(u)\right)(n+m-3)}}{n+m-2-d_{G}(u)} \\
& +\frac{m(m-1)}{2} .
\end{aligned}
$$

Proof. Partition the edge set $E\left(\overline{G^{++}}\right)$into three sets $E_{1}, E_{2}$ and $E_{3}$, where $E_{1}=\{u v \mid u v \notin E(G)\}$,

$$
E_{2}=\{u e \mid \text { the vertex } u \text { is not incident to the edge } e \text { in } G\}
$$

and $E_{3}=\{e f \mid e, f \in E(G)\}$. It is easy to check that $\left|E_{1}\right|=\left(\begin{array}{c}n \\ 2\end{array}\right)-m,\left|E_{2}\right|=$ $m(n-2)$ and $E_{3}=\left(\begin{array}{c}m \\ 2\end{array}\right)$. By Proposition 2.2 , if $u \in V(G)$ then $d_{\overline{G^{++}}}(u)=$ $n+m-1-2 d_{G}(u)$ and if $e \in E(G)$ then $d_{\overline{G^{++}}}(e)=n+m-3$.

Therefore

$$
\begin{aligned}
G A\left(\overline{G^{++}}\right) & =\sum_{u v \in E\left(\overline{G^{++}}\right)} \frac{\sqrt{d \overline{G^{++}}}(u) d \overline{G^{++}}(v)}{\frac{1}{2}\left[d \overline{G^{++}}(u)+d \overline{G^{++}}(v)\right]} \\
& =\sum_{u v \in E_{1}} \frac{\sqrt{d \overline{G^{++}}}(u) d \overline{G^{++}}(v)}{\frac{1}{2}\left[d \overline{G^{++}}(u)+d \overline{G^{++}}(v)\right]}
\end{aligned}
$$




$$
\begin{aligned}
& +\sum_{u e \in E_{2}} \frac{\sqrt{d_{\overline{G^{++}}}}(u) d \overline{G^{++}}(e)}{\frac{1}{2}\left[d_{\overline{G^{++}}}(u)+d_{\overline{G^{++}}}(e)\right]} \\
& +\sum_{e f \in E_{3}} \frac{\sqrt{d_{\overline{G^{++}}}}(e) d \overline{G^{++}}(f)}{\frac{1}{2}\left[d_{\overline{G^{++}}}(e)+d_{\overline{G^{++}}}(f)\right]} \\
& =\sum_{u v \notin E(G)} \frac{\sqrt{\left(n+m-1-2 d_{G}(u)\right)\left(n+m-1-2 d_{G}(v)\right)}}{\frac{1}{2}\left[n+m-1-2 d_{G}(u)+n+m-1-2 d_{G}(v)\right]} \\
& +\sum_{u e \in E_{2}} \frac{\sqrt{\left(n+m-1-2 d_{G}(u)\right)(n+m-3)}}{\frac{1}{2}\left[n+m-1-2 d_{G}(u)+n+m-3\right]} \\
& +\sum_{e f \in E_{3}} \frac{\sqrt{(n+m-3)(n+m-3)}}{\frac{1}{2}[n+m-3+n+m-3]} \\
& =\sum_{u v \notin E(G)} \frac{\sqrt{\left(n+m-1-2 d_{G}(u)\right)\left(n+m-1-2 d_{G}(v)\right)}}{n+m-1-\left(d_{G}(u)+d_{G}(v)\right)} \\
& +\sum_{u e \in E_{2}} \frac{\sqrt{\left(n+m-2-d_{G}(u)\right)(n+m-3)}}{n+m-2-d_{G}(u)}+\sum_{e f \in E_{3}} \\
& =\sum_{u v \notin E(G)} \frac{\sqrt{\left(n+m-1-2 d_{G}(u)\right)\left(n+m-1-2 d_{G}(v)\right)}}{n+m-1-\left(d_{G}(u)+d_{G}(v)\right)} \\
& +\sum_{u \in V(G)} \frac{\left(m-d_{G}(u)\right) \sqrt{\left(n+m-2-d_{G}(u)\right)(n+m-3)}}{n+m-2-d_{G}(u)} \\
& +\frac{m(m-1)}{2} \text {. }
\end{aligned}
$$

Theorem 4.2. Let $G$ be a graph with $n$ vertices and $m$ edges. Then

$$
G A\left(\overline{G^{+-}}\right)=\frac{n(n-1)-2 m}{2}+\frac{4 m \sqrt{(n-1)(m+1)}}{(n+m)}+\frac{m(m-1)}{2} .
$$

Proof. Partition the edge set $E\left(\overline{G^{+-}}\right)$into three sets $E_{1}, E_{2}$ and $E_{3}$, where $E_{1}=\{u v \mid u v \notin E(G)\}, E_{2}=\{u e \mid$ the vertex $u$ is incident to the edge $e$ in $G\}$ and $E_{3}=\{e f \mid e, f \in E(G)\}$. It is easy to check that $\left|E_{1}\right|=\left(\begin{array}{l}n \\ 2\end{array}\right)-m,\left|E_{2}\right|=2 m$ and $E_{3}=\left(\begin{array}{c}m \\ 2\end{array}\right)$. By Proposition 2.2, if $u \in V(G)$ then $d_{\overline{G^{+-}}}(u)=n-1$ and if $e \in E(G)$ then $d_{\overline{G^{+-}}}(e)=m+1$. 
Therefore

$$
\begin{aligned}
& G A\left(\overline{G^{+-}}\right)=\sum_{u v \in E\left(\overline{G^{+-}}\right)} \frac{\sqrt{d_{\overline{G^{+-}}}(u) d \overline{G^{+-}}(v)}}{\frac{1}{2}\left[d_{\overline{G^{+-}}}(u)+d_{\overline{G^{+-}}}(v)\right]} \\
& =\sum_{u v \in E_{1}} \frac{\sqrt{d \overline{G^{+-}}}(u) d \overline{G^{+-}}(v)}{\frac{1}{2}\left[d \overline{G^{+-}}(u)+d \overline{G^{+-}}(v)\right]}+\sum_{u e \in E_{2}} \frac{\sqrt{d \overline{G^{+-}}}(u) d_{\overline{G^{+-}}}(e)}{\frac{1}{2}\left[d \overline{G^{+-}}(u)+d \overline{G^{+-}}(e)\right]} \\
& +\sum_{e f \in E_{3}} \frac{\sqrt{d \overline{G^{+-}}}(e) d \overline{G^{+-}}(f)}{\frac{1}{2}\left[d \overline{G^{+-}}(e)+d \overline{G^{+-}}(f)\right]} \\
& =\sum_{u v \notin E(G)} \frac{\sqrt{(n-1)(n-1)}}{\frac{1}{2}[n-1+n-1]}+\sum_{u \in \in E_{2}} \frac{\sqrt{(n-1)(m+1)}}{\frac{1}{2}[n-1+m+1]} \\
& +\sum_{e f \in E_{3}} \frac{\sqrt{(m+1)(m+1)}}{\frac{1}{2}[m+1+m+1]} \\
& =\sum_{u v \notin E(G)}(1)+\sum_{u e \in E_{2}} \frac{\sqrt{(n-1)(m+1)}}{\frac{1}{2}[n+m]}+\sum_{e f \in E_{3}}(1) \\
& =\left[\left(\begin{array}{l}
n \\
2
\end{array}\right)-m\right]+\frac{2 m \sqrt{(n-1)(m+1)}}{\frac{1}{2}(n+m)}+\left(\begin{array}{c}
m \\
2
\end{array}\right) \\
& =\frac{n(n-1)-2 m}{2}+\frac{4 m \sqrt{(n-1)(m+1)}}{(n+m)}+\frac{m(m-1)}{2} \text {. }
\end{aligned}
$$

Theorem 4.3. Let $G$ be a graph with $n$ vertices and $m$ edges. Then

$$
G A\left(\overline{G^{-+}}\right)=m+\frac{2 m(n-2) \sqrt{m(n+m-3)}}{(2 m+n-3)}+\frac{m(m-1)}{2} .
$$

Proof. Partition the edge set $E\left(\overline{G^{-+}}\right)$into three sets $E_{1}, E_{2}$ and $E_{3}$, where $E_{1}=\{u v \mid u v \in E(G)\}$,

$$
E_{2}=\{u e \mid \text { the vertex } u \text { is not incident to the edge } e \text { in } G\}
$$

and $E_{3}=\{e f \mid e, f \in E(G)\}$. It is easy to check that $\left|E_{1}\right|=m,\left|E_{2}\right|=m(n-2)$ and $E_{3}=\left(\begin{array}{c}m \\ 2\end{array}\right)$. By Proposition 2.2, if $u \in V(G)$ then $d_{\overline{G^{-+}}}(u)=m$ and if $e \in E(G)$ then $d \overline{G^{-+}}(e)=n+m-3$.

Therefore

$$
G A\left(\overline{G^{-+}}\right)=\sum_{u v \in E\left(\overline{G^{-+}}\right)} \frac{\sqrt{d \overline{G^{-+}}(u) d \overline{G^{-+}}(v)}}{\frac{1}{2}\left[d \overline{G^{-+}}(u)+d \overline{G^{-+}}(v)\right]}
$$




$$
\begin{aligned}
= & \sum_{u v \in E_{1}} \frac{\sqrt{d_{\overline{G^{-+}}}}(u) d_{\overline{G^{-+}}}(v)}{\frac{1}{2}\left[d_{\overline{G^{-+}}}(u)+d_{\overline{G^{-+}}}(v)\right]}+\sum_{u e \in E_{2}} \frac{\sqrt{d_{\overline{G^{-+}}}}(u) d_{\overline{G^{-+}}}(e)}{\frac{1}{2}\left[d_{\overline{G^{-+}}}(u)+d_{\overline{G^{-+}}}(e)\right]} \\
& +\sum_{e f \in E_{3}} \frac{\sqrt{d_{\overline{G^{-+}}}}(e) d_{\overline{G^{-+}}}(f)}{\frac{1}{2}\left[d_{\overline{G^{-+}}}(e)+d_{\overline{G^{-+}}}(f)\right]} \\
= & \sum_{u v \in E(G)} \frac{\sqrt{(m)(m)}}{\frac{1}{2}[m+m]}+\sum_{u e \in E_{2}} \frac{\sqrt{m(n+m-3)}}{\frac{1}{2}[m+n+m-3]} \\
= & \sum_{e f \in E_{3}} \frac{\sqrt{(n+m-3)(n+m-3)}}{\frac{1}{2}[n+m-3+n+m-3]} \\
= & m+\frac{2 m(1)+\sum_{u v \in E} \frac{2 \sqrt{m(n+m-3)}}{(2 m+n-3)}+\sum_{e f \in E_{3}}(1)}{(2 m+n-3)}+\frac{m(m-1)}{2} .
\end{aligned}
$$

Theorem 4.4. Let $G$ be a graph with $n$ vertices and $m$ edges. Then

$$
G A\left(\overline{G^{--}}\right)=G A(G)+\sum_{u \in V(G)} \frac{2 d_{G}(u) \sqrt{\left(2 d_{G}(u)\right)(m+1)}}{\left(2 d_{G}(u)+m+1\right)}+\frac{m(m-1)}{2} .
$$

Proof. Partition the edge set $E\left(\overline{G^{--}}\right)$into three sets $E_{1}, E_{2}$ and $E_{3}$, where $E_{1}=\{u v \mid u v \in E(G)\}, E_{2}=\{u e \mid$ the vertex $u$ is incident to the edge $e$ in $G\}$ and $E_{3}=\{e f \mid e, f \in E(G)\}$. It is easy to check that $\left|E_{1}\right|=m,\left|E_{2}\right|=2 m$ and $E_{3}=\left(\begin{array}{c}m \\ 2\end{array}\right)$. By Proposition 2.2, if $u \in V(G)$ then $d_{\overline{G^{--}}}(u)=2 d_{G}(u)$ and if $e \in E(G)$ then $d_{\overline{G^{--}}}(e)=m+1$.

Therefore

$$
\begin{aligned}
& G A\left(\overline{G^{--}}\right)=\sum_{u v \in E\left(\overline{G^{--}}\right)} \frac{\sqrt{d \overline{G^{--}}(u) d \overline{G^{--}}(v)}}{\frac{1}{2}\left[d \overline{G^{--}}(u)+d \overline{G^{--}}(v)\right]} \\
& =\sum_{u v \in E_{1}} \frac{\sqrt{d \overline{G^{--}}}(u) d \overline{G^{--}}(v)}{\frac{1}{2}\left[d_{\overline{G^{--}}}(u)+d_{\overline{G^{--}}}(v)\right]}+\sum_{u \in \in E_{2}} \frac{\sqrt{d_{\overline{G^{--}}}}(u) d_{\overline{G^{--}}}(e)}{\frac{1}{2}\left[d_{\overline{G^{-}}}(u)+d_{\overline{G^{--}}}(e)\right]} \\
& +\sum_{e f \in E_{3}} \frac{\sqrt{d \overline{G^{--}}}(e) d \overline{G^{--}}(f)}{\frac{1}{2}\left[d \overline{G^{--}}(e)+d \overline{G^{--}}(f)\right]}
\end{aligned}
$$




$$
\begin{aligned}
= & \sum_{u v \in E(G)} \frac{\sqrt{\left(2 d_{G}(u)\right)\left(2 d_{G}(v)\right)}}{\frac{1}{2}\left[2 d_{G}(u)+2 d_{G}(v)\right]}+\sum_{u e \in E_{2}} \frac{\sqrt{\left(2 d_{G}(u)\right)(m+1)}}{\frac{1}{2}\left[2 d_{G}(u)+m+1\right]} \\
& +\sum_{e f \in E_{3}} \frac{\sqrt{(m+1)(m+1)}}{\frac{1}{2}[(m+1)+(m+1)]} \\
= & \sum_{u v \in E(G)} \frac{\sqrt{\left(d_{G}(u)\right)\left(d_{G}(v)\right)}}{\frac{1}{2}\left[d_{G}(u)+d_{G}(v)\right]} \\
& +\sum_{u e \in E_{2}} \frac{2 \sqrt{\left(2 d_{G}(u)\right)(m+1)}}{\left(2 d_{G}(u)+m+1\right)}+\sum_{e f \in E_{3}}(1) \\
= & G A(G)+\sum_{u \in V(G)} \frac{2 d_{G}(u) \sqrt{\left(2 d_{G}(u)\right)(m+1)}}{\left(2 d_{G}(u)+m+1\right)}+\frac{m(m-1)}{2} .
\end{aligned}
$$

By Theorems 4.2 and 4.3 we have following corollary.

Corollary 4.5. Let $G_{1}$ and $G_{2}$ be two different graphs having same number of vertices and same number of edges. Then

(i) $G A\left(\overline{G_{1}^{+-}}\right)=G A\left(\overline{G_{2}^{+-}}\right)$

(ii) $G A\left(\overline{G_{1}^{-+}}\right)=G A\left(\overline{G_{2}^{-+}}\right)$.

\section{Sum-Connectivity Index, Augmented Zagreb Index and Atom-Bond Connectivity Index of $G^{x y}$ and $\overline{G^{x y}}$}

In fully analogous manner, applying the proof techniques of the Sections 3 and 4 we arrive at the results of this section.

Theorem 5.1. Let $G$ be a graph with $n$ vertices and $m$ edges. Then

$$
\begin{aligned}
& \text { (i) } S C\left(G^{++}\right)=\frac{1}{\sqrt{2}}\left[S C(G)+\sum_{u \in V(G)} \frac{d_{G}(u)}{\sqrt{1+d_{G}(u)}}\right] \\
& \text { (ii) } S C\left(G^{+-}\right)=\sqrt{\frac{m}{2}}+\frac{m(n-2)}{\sqrt{m+n-2}} \\
& \text { (iii) } S C\left(G^{-+}\right)=\frac{n(n-1)-2 m}{2 \sqrt{2 n-2}}+\frac{2 m}{\sqrt{n+1}} \\
& \text { (iv) } S C\left(G^{--}\right)=\sum_{u v \notin E(G)} \frac{1}{\sqrt{2\left(n+m-1-d_{G}(u)-d_{G}(v)\right)}}
\end{aligned}
$$




$$
+\sum_{u \in V(G)} \frac{m-d_{G}(u)}{\sqrt{2 n+m-3-2 d_{G}(u)}}
$$

(v) $S C\left(\overline{G^{++}}\right)=\sum_{u v \notin E(G)} \frac{1}{\sqrt{2\left(n+m-1-d_{G}(u)-d_{G}(v)\right)}}$

$$
+\sum_{u \in V(G)} \frac{m-d_{G}(u)}{\sqrt{2\left(n+m-2-d_{G}(u)\right)}}+\frac{m(m-1)}{2 \sqrt{2(n+m-3)}}
$$

(vi) $S C\left(\overline{G^{+-}}\right)=\frac{n(n-1)-2 m}{2 \sqrt{(2(n-1))}}+\frac{2 m}{\sqrt{n+m}}+\frac{m(m-1)}{2 \sqrt{2(m+1)}}$

(vii) $S C\left(\overline{G^{-+}}\right)=\sqrt{\frac{m}{2}}+\frac{m(n-2)}{\sqrt{2 m+n-3}}+\frac{m(m-1)}{2 \sqrt{2(n+m-3)}}$

(viii) $S C\left(\overline{G^{--}}\right)=\frac{1}{\sqrt{2}} S C(G)+\frac{m(m-1)}{2 \sqrt{2(m+1)}}+\sum_{u \in V(G)} \frac{d_{G}(u)}{\sqrt{m+1+2 d_{G}(u)}}$.

Corollary 5.2. Let $G_{1}$ and $G_{2}$ be two different graphs having same number of vertices and same number of edges. Then

(i) $S C\left(G_{1}^{+-}\right)=S C\left(G_{2}^{+-}\right)$

(ii) $S C\left(G_{1}^{-+}\right)=S C\left(G_{2}^{-+}\right)$

(iii) $S C\left(\overline{G_{1}^{+-}}\right)=S C\left(\overline{G_{2}^{+-}}\right)$

(iv) $S C\left(\overline{G_{1}^{-+}}\right)=S C\left(\overline{G_{2}^{-+}}\right)$.

Theorem 5.3. Let $G$ be a graph with $n$ vertices and $m$ edges. Then

$$
\begin{aligned}
\text { (i) } A Z\left(G^{++}\right)= & 16 m+\sum_{u v \in E(G}\left[\frac{2\left(d_{G}(u)\right)\left(d_{G}(v)\right)}{d_{G}(u)+d_{G}(v)-1}\right]^{3} \\
\text { (ii) } A Z\left(G^{+-}\right)= & \frac{m^{7}}{(2 m-2)^{3}}+\frac{(m(n-2))^{4}}{(m+n-4)^{3}} \\
\text { (iii) } A Z\left(G^{-+}\right)= & {\left[\frac{n(n-1)-2 m}{2}\right]\left[\frac{(n-1)^{6}}{(2 n-4)^{3}}\right]+16 m } \\
\text { (iv) } A Z\left(G^{--}\right)= & \sum_{u v \notin E(G)}\left[\frac{\left(n+m-1-2 d_{G}(u)\right)\left(n+m-1-2 d_{G}(v)\right)}{2\left(n+m-2-d_{G}(u)-d_{G}(v)\right)}\right]^{3} \\
& +\sum_{u \in V(G)}\left(m-d_{G}(u)\right)\left[\frac{\left(n+m-1-2 d_{G}(u)\right)(n-2)}{2 n+m-5-2 d_{G}(u)}\right]^{3} \\
\text { (v) } A Z\left(\overline{G^{++}}\right)= & \sum_{u v \notin E(G)}\left[\frac{\left(n+m-1-2 d_{G}(u)\right)\left(n+m-1-2 d_{G}(v)\right)}{2\left(n+m-2-d_{G}(u)-d_{G}(v)\right)}\right]^{3}
\end{aligned}
$$




$$
\begin{aligned}
& +\sum_{u \in V(G)}\left(m-d_{G}(u)\right)\left[\frac{\left(n+m-1-2 d_{G}(u)\right)(n+m-3]^{3}}{2\left(n+m-3-d_{G}(u)\right)}\right]^{3} \\
& +\frac{m(m-1)}{2}\left[\frac{(n+m-3)^{6}}{8(n+m-4)^{3}}\right] \\
\text { (vi) } A Z\left(\overline{G^{+-}}\right)= & {\left[\frac{n(n-1)-2 m}{2}\left[\frac{(n-1)^{6}}{(2 n-4)^{3}}\right]+2 m\left[\frac{(n-1)(m+1)}{n+m-2}\right]^{3}\right.} \\
& +\frac{(m-1)(m+1)^{6}}{16 m^{2}} \\
\text { (vii) } A Z\left(\overline{G^{-+}}\right)= & \frac{m^{7}}{8(m-1)^{3}}+\frac{m^{4}(n-2)(n+m-3)^{3}}{n+2 m-5} \\
& +\frac{m(m-1)(n+m-3)^{6}}{16(n+m-4)^{3}} \\
\text { (viii) } A Z\left(\overline{G^{--}}\right)= & \sum_{u v E(G)}\left[\frac{2 d_{G}(u) d_{G}(v)}{d_{G}(u)+d_{G}(v)-1}\right]^{3}+\frac{(m-1)(m+1)^{6}}{16 m^{2}} \\
& +\sum_{u \in V(G)} \frac{8(m+1)^{3}\left(d_{G}(u)\right)^{4}}{\left(2 d_{G}(u)+m-1\right)^{3}} .
\end{aligned}
$$

Corollary 5.4. Let $G_{1}$ and $G_{2}$ be two different graphs having same number of vertices and same number of edges. Then

(i) $A Z\left(G_{1}^{+-}\right)=A Z\left(G_{2}^{+-}\right)$

(ii) $A Z\left(G_{1}^{-+}\right)=A Z\left(G_{2}^{-+}\right)$

(iii) $A Z\left(\overline{G_{1}^{+-}}\right)=A Z\left(\overline{G_{2}^{+-}}\right)$

(iv) $A Z\left(\overline{G_{1}^{-+}}\right)=A Z\left(\overline{G_{2}^{-+}}\right)$.

Theorem 5.5. Let $G$ be a graph with $n$ vertices and $m$ edges. Then:

$$
\begin{aligned}
\text { (i) } A B C\left(G^{++}\right) & =\sqrt{2} m+\sum_{u v \in E(G}\left[\frac{d_{G}(u)+d_{G}(v)-1}{2\left(d_{G}(u)\right)\left(d_{G}(v)\right)}\right]^{1 / 2} \\
\text { (ii) } A B C\left(G^{+-}\right) & =\sqrt{2 m-2}+\sqrt{m(n-2)(m+n-4)} \\
\text { (iii) } A B C\left(G^{-+}\right) & =\left[\frac{n(n-1)-2 m}{2}\right]\left[\frac{\sqrt{2 n-4}}{n-1}\right]+\sqrt{2} m \\
\text { (iv) } A B C\left(G^{--}\right) & =\sum_{u v \notin E(G)}\left[\frac{2\left(n+m-2-d_{G}(u)-d_{G}(v)\right)}{\left(n+m-1-2 d_{G}(u)\right)\left(n+m-1-2 d_{G}(v)\right)}\right]^{1 / 2}
\end{aligned}
$$




$$
\begin{aligned}
& +\sum_{u \in V(G)}\left(m-d_{G}(u)\right)\left[\frac{2 n+m-5-2 d_{G}(u)}{\left(n+m-1-2 d_{G}(u)\right)(n-2)}\right]^{1 / 2} \\
& \text { (v) } A B C\left(\overline{G^{++}}\right)=\sum_{u v \notin E(G)}\left[\frac{2\left(n+m-2-d_{G}(u)-d_{G}(v)\right)}{\left(n+m-1-2 d_{G}(u)\right)\left(n+m-1-2 d_{G}(v)\right)}\right]^{1 / 2} \\
& +\sum_{u \in V(G)}\left(m-d_{G}(u)\right)\left[\frac{2\left(n+m-3-d_{G}(u)\right)}{\left(n+m-1-2 d_{G}(u)\right)(n+m-3}\right]^{1 / 2} \\
& +\frac{m(m-1) \sqrt{n+m-4}}{\sqrt{2}(n+m-3)} \\
& \text { (vi) } A B C\left(\overline{G^{+-}}\right)=\left[\frac{n(n-1)-2 m}{2}\right]\left[\frac{\sqrt{2 n-4}}{n-1}\right]+\frac{2 m \sqrt{n+m-2}}{(n-1)(m+1)} \\
& +\frac{m^{3 / 2}(m-1)}{\sqrt{2}(m+1)} \\
& \text { (vii) } A B C\left(\overline{G^{-+}}\right)=\left[\frac{2(m-1)}{m}\right]^{1 / 2}+(n-2)\left[\frac{m(n+2 m-5)}{n+m-3}\right]^{1 / 2} \\
& +\frac{m(m-1) \sqrt{n+m-4}}{\sqrt{2}(n+m-3)} \\
& \text { (viii) } A B C\left(\overline{G^{--}}\right)=\sum_{u v \in E(G)}\left[\frac{d_{G}(u)+d_{G}(v)-1}{2 d_{G}(u) d_{G}(v)}\right]^{1 / 2} \\
& +\sum_{u \in V(G)}\left[\frac{\left(d_{G}(u)\right)\left(2 d_{G}(u)+m-1\right)}{2(m+1)}\right]^{1 / 2} \\
& +\frac{m^{3 / 2} \sqrt{m-1}}{\sqrt{2}(m+1)}
\end{aligned}
$$

Corollary 5.6. Let $G_{1}$ and $G_{2}$ be two different graphs having same number of vertices and same number of edges. Then
(i) $A B C\left(G_{1}^{+-}\right)=A B C\left(G_{2}^{+-}\right)$
(ii) $A B C\left(G_{1}^{-+}\right)=A B C\left(G_{2}^{-+}\right)$
(iii) $A B C\left(\overline{G_{1}^{+-}}\right)=A B C\left(\overline{G_{2}^{+-}}\right)$
(iv) $A B C\left(\overline{G_{1}^{-+}}\right)=A B C\left(\overline{G_{2}^{-+}}\right)$. 


\section{Acknowledgments}

The authors H. S. Ramane and R. B. Jummannaver are thankful to the University Grants Commission (UGC), Govt. of India for support through research grant under UPE FAR-II grant No. F 14-3/2012 (NS/PE).

\section{References}

[1] B. Basavanagoud, I. Gutman, V. R. Desai, Zagreb indices of generalized transformation graphs and their complements, Kragujevac J. Sci., 37 (2015), 99-112.

[2] K. C. Das, On comparing Zagreb index of graphs, MATCH Commun. Math. Comput. Chem., 63 (2010), 433-440.

[3] E. Estrada, Atom-bond connectivity and the energetic of branched alkanes, Chem. Phys. Lett., 463 (2008), 422-425.

[4] E. Estrada, L. Torres, L. Rodríguez, I. Gutman, An atom-bond connectivity index: Modelling the enthalpy of formation of alkanes, Indian J. Chem., 37A (1998), 849-855.

[5] S. Fajtlowicz, On conjectures of Graffiti - II, Congr. Numer., 60 (1987), 187-197.

[6] B. Furtula, A. Graovac, D. Vukicević, Augmented Zagreb index, J. Math. Chem., 48 (2010), 370-380.

[7] I. Gutman, K.C. Das, The first Zagreb index 30 years after, MATCH Commun. Math. Comput. Chem., 50 (2004), 83-92.

[8] I. Gutman, N. Trinajstić, Graph theory and molecular orbitals, Total $\pi$-electron energy of alternant hydrocarbons, Chem. Phys. Lett., 17 (1972), 535-538.

[9] X. Li, Y. Shi, A survey on the Randić index, MATCH Commun. Math. Comput. Chem., 59 (2008), 127-156.

[10] J. Li, W. C. Shiu, The harmonic index of a graph, Rocky Mountain J. Math., 44 (2014), 1607-1620.

[11] B. Lučić, S. Nikolić, N. Trinajstić, B. Zhou, S. Ivaniš Turk, Sum-connectivity index, in: I. Gutman, B. Furtula (Eds.), Novel Molecular Structure Descriptors - Theory and Applications I, Kragujevac Univ., Kragujevac, 2010, pp. 101-136.

[12] B. Lučić, N. Trinajstić, B. Zhou, Comparison between the sum-connectivity index and product-connectivity index for benzenoid hydrocarbons, Chem. Phys. Lett., 475 (2009), 146-148.

[13] H. S. Ramane, B. Basavanagoud, R. B. Jummannaver, Harmonic index and Randić index of generalized transformation graphs, preprint.

[14] M. Randić, On characterization of molecular branching, J. Am. Chem. Soc., 97 (1975), 6609-6615.

[15] E. Sampathkumar, S. B. Chikkodimath, Semitotal graphs of a graph - I, J. Karnatak Univ. Sci., 18 (1973), 274-280.

[16] D. Vukičević, B. Furtula, Topological index based on the ratios of geometrical and arithmetical means of end-vertex degrees of edges, J. Math. Chem., 46 (2009), 1369-1376. 
[17] B. Zhou, N. Trinajstić, On a novel connectivity index, J. Math. Chem., 46 (2009), 12521270. 
